\title{
TeV dark matter and the DAMPE electron excess
}

\author{
Xuewen $\mathrm{Liu}^{1, *}$ and Zuowei $\mathrm{Liu}^{1,2,3, \dagger}$ \\ ${ }^{1}$ Department of Physics, Nanjing University, Nanjing 210093, China \\ ${ }^{2}$ Center for High Energy Physics, Peking University, Beijing 100871, China \\ ${ }^{3}$ CAS Center for Excellence in Particle Physics, Beijing 100049, China
}

(Received 4 December 2017; published 16 August 2018)

\begin{abstract}
The recent high energy electron and positron flux observed by the DAMPE experiment indicates possible excess events near $1.4 \mathrm{TeV}$. Such an excess may be evidence of dark matter annihilations or decays in a dark matter subhalo that is located close to the solar system. We give here an analysis of this excess from annihilations of Dirac fermion dark matter which is charged under a new $U(1)_{X}$ gauge symmetry. The interactions between dark matter and the standard model particles are mediated by the $U(1)_{X}$ gauge boson. We show that dark matter annihilations from a local subhalo can explain the excess with the canonical thermal annihilation cross section. We further discuss the constraints from the relic density, from the dark matter direct detection, from the dark matter indirect detection, from the cosmic microwave background, and from the particle colliders.
\end{abstract}

DOI: $10.1103 /$ PhysRevD.98.035025

\section{INTRODUCTION}

Recently, evidence for excess electron and positron events near $1.4 \mathrm{TeV}$ has been reported by the DAMPE experiment [1]. Such an excess was not found previously by the AMS-02 [2-4] and by the Fermi-LAT [5]. In the recent paper by the CALET experiment [6], the electron and positron events in the two energy bins near $1 \mathrm{TeV}$ also appear higher than expected. Due to the better energy resolution of the DAMPE experiment $(\sim 1 \%$ for $1 \mathrm{TeV}$ electrons and positrons [7]) than AMS-02, Fermi-LAT, and CALET, the $\mathrm{TeV}$ electron and positron flux can be measured with better accuracy by the DAMPE experiment than measured previously by other experiments. In the DAMPE data, the electron and positron excess events occur only in one energy bin near $1.4 \mathrm{TeV}$. The localized feature in the energy spectrum of the excess events hints a nearby source of the high energy electrons and positrons. Inspired by this excess, studies with dark matter (DM) explanation [8-11] and with astrophysical explanation [11,12] have been carried out. In this paper, we study the possibility of attributing the excess of the $\mathrm{TeV}$ electrons and positrons to the DM annihilations in the vicinity of the solar system.

\footnotetext{
*xuewenliu@nju.edu.cn tzuoweiliu@nju.edu.cn
}

Published by the American Physical Society under the terms of the Creative Commons Attribution 4.0 International license. Further distribution of this work must maintain attribution to the author(s) and the published article's title, journal citation, and DOI. Funded by SCOAP ${ }^{3}$.

\section{THE MODEL}

We consider a $U(1)_{X}$ extension of the standard model (SM) with $X_{\mu}$ as the new gauge boson, and $\chi$ as the Dirac DM particle which is charged under the $U(1)_{X}$ gauge symmetry. The new Lagrangian terms are

$$
\mathcal{L}=-\frac{1}{4} X_{\mu \nu} X^{\mu \nu}-\frac{1}{2} M_{X}^{2} X_{\mu} X^{\mu}+X_{\mu} J^{\mu},
$$

where $X_{\mu}$ is the new gauge boson, $X_{\mu \nu}$ is the field strength, $M_{X}$ is the $X$ boson mass. The $X$ boson is assumed to couple to the SM fermions and to the Dirac DM in the vector current form, $J_{\mu}=g_{f} \bar{f} \gamma_{\mu} f+g_{\chi} \bar{\chi} \gamma_{\mu} \chi$, where $f$ stands for the SM fermion.

In this paper, we consider three scenarios: (A) DM annihilates into $e^{+} e^{-}$only via an s-channel exchange of the $X_{\mu}$ boson (assuming only $g_{e} \neq 0$ ); (B) DM annihilates into a pair of on-shell $X$ bosons by exchanging a t-channel or a u-channel DM fermion, and the $X$ boson subsequently decays into $e^{+} e^{-}$; (C) DM annihilates into all SM fermion final states universally via the s-channel $X_{\mu}$ boson.

The annihilation cross section for the $\chi \chi \rightarrow X \rightarrow \bar{f} f$ process is given by

$$
\sigma v=\frac{N_{f} g_{\chi}^{2} g_{f}^{2}}{6 \pi} \frac{s+2 m_{\chi}^{2}}{\left(s-M_{X}^{2}\right)^{2}+M_{X}^{2} \Gamma_{X}^{2}}
$$

where $N_{f}=1$ (3) for leptons (quarks), $\Gamma_{X}$ is the total decay width of the $X$ boson, and we have neglected the mass of the final state SM particles. The partial decay width of the $X$ boson is $\Gamma(X \rightarrow \bar{f} f)=N_{f} g_{f}^{2} M_{X} /(12 \pi)$. If the DM 
annihilation occurs away from the $X$ resonance and the $X$ boson has a narrow decay width, i.e., $\Gamma_{X} \ll M_{X}$, one has $\sigma v \simeq N_{c}^{f} g_{f}^{2} g_{\chi}^{2} /\left(m_{\chi}^{2} \pi\left(x^{2}-4\right)^{2}\right)$ where $x \equiv M_{X} / m_{\chi}$. For the case in which the $X$ boson only couples to electrons, in order to obtain the desired $1 \mathrm{pb}$ annihilation cross section, one has $\sqrt{\left|g_{e} g_{\chi}\right|} \simeq 0.37 \sqrt{\left|x^{2}-4\right|}$ where $x=M_{X} /(1.5 \mathrm{TeV})$, if the DM annihilation occurs away from the $X$ resonance.

\section{COSMIC RAY PROPAGATION}

The propagation of the electrons and positrons can be described by the following diffusion equation

$$
\partial_{t} f-\partial_{E}(b(E) f)-D(E) \nabla^{2} f=Q,
$$

where $f=d N /(d E d V)$ is the electron energy spectrum, $b(E)=-d E / d t$ is the energy loss coefficient, $D(E)$ is the diffusion coefficient, and $Q=Q(\mathbf{x}, E, t)$ is the source term. We take $b(E)=b_{0}(E / \mathrm{GeV})^{2}$ with $b_{0}=10^{-16} \mathrm{GeV} / \mathrm{s}$ and $D(E)=D_{0}(E / \mathrm{GeV})^{\delta}$. The diffusion coefficient $D(E)$ depends on the height $2 L$ in the $z$ direction of the cylindrical diffusion zone which is usually assumed for the Milky Way (MW) galaxy. We adopt the medium case in Ref. [13] such that $L=4 \mathrm{kpc}, D_{0}=11 \mathrm{pc}^{2} / \mathrm{kyr}$, and $\delta=0.7$. For the steady-state case, the Green's function of the diffusion equation is given by [14-16]

$$
G\left(\mathbf{x}, E ; \mathbf{x}_{s}, E_{s}\right)=\frac{\exp \left[-\left(\mathbf{x}-\mathbf{x}_{s}\right)^{2} / \lambda^{2}\right]}{b(E)\left(\pi \lambda^{2}\right)^{3 / 2}},
$$

where $\lambda$ is the propagation scale which is given by

$$
\lambda^{2}=4 \int_{E}^{E_{s}} d E^{\prime} \frac{D\left(E^{\prime}\right)}{b\left(E^{\prime}\right)} .
$$

By using the Green's function, the general solution to the diffusion equation can be computed via

$f(\mathbf{x}, E)=\int d^{3} x_{s} \int d E_{s} G\left(\mathbf{x}, E ; \mathbf{x}_{s}, E_{s}\right) Q\left(\mathbf{x}_{s}, E_{s}\right)$.

For DM annihilations, the source function of electrons and positrons is given by

$$
Q(\mathbf{x}, E)=\frac{1}{4} \frac{\rho_{\chi}^{2}(\mathbf{x})}{m_{\chi}^{2}}\langle\sigma v\rangle \frac{d N}{d E},
$$

where $\rho_{\chi}(\mathbf{x})$ is the DM mass density, $m_{\chi}$ is the DM mass, $\langle\sigma v\rangle$ is the velocity-averaged annihilation cross section, $\frac{d N}{d E}$ is the energy spectrum of electrons and positrons per annihilation, and the $1 / 4$ factor is due to the Dirac nature of the DM (1/2 for Majorana DM). The electron and positron flux per unit energy from DM annihilations is given by $\Phi(\mathbf{x}, E)=v f(\mathbf{x}, E) /(4 \pi)$ where $v$ is the electron velocity; the unit of the flux is $1 /\left(\mathrm{GeV} \cdot \mathrm{cm}^{2} \cdot \mathrm{s} \cdot \mathrm{sr}\right)$ [13].

\section{ELECTRON FLUX FROM A LOCAL SUBHALO}

We consider an ultra-faint DM subhalo which is located $\lesssim 1 \mathrm{kpc}$ away from us. We assume a NFW density profile [17] for the subhalo

$$
\rho(r)=\rho_{s} \frac{\left(r / r_{s}\right)^{-\gamma}}{\left(1+r / r_{s}\right)^{3-\gamma}} .
$$

The distance between the subhalo and us is denoted as $d_{s}$ which is taken to be in the range $(0.1-1) \mathrm{kpc}$. In the following we consider two different sets of parameters $\left(\gamma, \rho_{s}, r_{s}, d_{s}\right)=(1,1,1,1)$ (denoted as SHA) and $(0.5,100,0.1,0.3)$ (denoted as SHB) where $\rho_{s}$ is in $\mathrm{GeV} / \mathrm{cm}^{3}$, and $r_{s}$ and $d_{s}$ are in $\mathrm{kpc}[18-20]$.

If two DM particles with $1.5 \mathrm{TeV}$ mass annihilate in the subhalo via $\chi \chi \rightarrow e^{+} e^{-}$only with $\sigma v=3 \times 10^{-26} \mathrm{~cm}^{3} / \mathrm{s}$, the electron flux at $E=1.4 \mathrm{TeV}$ is $E^{3} \Psi_{\chi}=0.2(47) \mathrm{GeV}^{2} /$ $\left(\mathrm{m}^{2} \cdot \mathrm{s} \cdot \mathrm{sr}\right)$ for the SHA (SHB) case. Thus, in order to produce the right amount of electrons and positrons observed by the DAMPE experiment [1], we will adopt the SHB assumption throughout the paper.

\section{COSMIC RAY BACKGROUND}

To understand the cosmic ray (CR) background is essential for astrophysical observations. To know the background predictions precisely in the measurement of the satellite experiments is the major challenge of the current DM indirect search experiments. Usually the CR background can be modeled via the broken power law (PL) forms. We adopt the parameterization formulas as in Ref. [21] where the background electrons and positrons consist of three components: the primary electrons from the CR sources, $\phi_{\text {primary, }}$ the secondary positrons/electrons originating from interactions between the primary CR and the interstellar medium, $\phi_{\text {secondary }}$, and the extra source, e.g., pulsars or DM, $\phi_{\text {source. }}$. For the primary electrons, the flux is parameterized as $\phi_{\text {primary }}=C E^{-\alpha} /\left(1+\left(E / E_{b}\right)^{\beta}\right)$; the secondary positron flux takes the same formula as the primary electron but with different coefficients. The extra source contains an exponential cutoff scale $E_{c}$, which takes the form $\phi_{\text {source }}=C E^{-\gamma} \exp \left(-E / E_{c}\right)$. The total flux for positrons is given by $\Phi_{e^{+}}=\phi_{\text {secondary }}+\phi_{\text {source, }}$, and the total flux for electrons is given by $\Phi_{e^{-}}=\phi_{\text {primary }}+0.6 \phi_{\text {secondary }}+\phi_{\text {source }}$ [21]. In our analysis, we used the electron plus positron flux measured by the DAMPE [1] to fit the various coefficients. The best-fit model is given as follows: for the primary electrons, $\left(C=16.67 \mathrm{GeV}^{2} \mathrm{~m}^{-2} \mathrm{~s}^{-1} \mathrm{sr}^{-1}, \alpha=\right.$ $1.20, \beta=2.10, E_{b}=4.98 \mathrm{GeV}$ ); for the secondary electron/positron flux, $\left(C=0.80 \mathrm{GeV}^{2} \mathrm{~m}^{-2} \mathrm{~s}^{-1} \mathrm{sr}^{-1}, \alpha=0.76\right.$, $\left.\beta=2.51, E_{b}=1.40 \mathrm{GeV}\right)$; for the additional source, $(C=$ $\left.0.99 \mathrm{GeV}^{2} \mathrm{~m}^{-2} \mathrm{~s}^{-1} \mathrm{sr}^{-1}, \gamma=2.32, E_{c}=686.75 \mathrm{GeV}\right)$. 


\section{DAMPE DATA FITTING}

We use $\Psi_{B}+\Psi_{\chi}$ to fit the DAMPE data [1], where $\Psi_{B}$ is the CR background, and $\Psi_{\chi}$ is the DM contribution from both the MW halo and the nearby subhalo. We carry out a $\chi^{2}$ analysis

$$
\chi^{2}=\sum_{i} \frac{\left(E_{i}^{3} \Phi_{i}^{\mathrm{th}}-E_{i}^{3} \Phi_{i}^{\mathrm{exp}}\right)^{2}}{\delta_{i}^{2}},
$$

where $\Phi_{i}^{\text {th }}=\Psi_{B}+\Psi_{\chi}$ is the predicted flux of electrons plus positrons in the bin $i, \Phi_{i}^{\exp }$ is the flux observed by the DAMPE experiment, and $\delta_{i}$ is the uncertainties reported by the DAMPE experiment [1]. For the DM signal, we use PPPC4DMID $[22,23]$ to generate the energy spectrum for the source function.

Figure 1 shows the DAMPE data [1] and various contributions to the electron flux. Here we analyze the $\chi \chi \rightarrow X \rightarrow e^{-} e^{+}$annihilation channel only, and a delta function energy spectrum $d N / d E=2 \delta\left(E-m_{\chi}\right)$ is employed for the injection source. The DM annihilation cross section is taken to be $\sigma v=3 \times 10^{-26} \mathrm{~cm}^{3} / \mathrm{s}$ and mass $m_{\chi}=1.5 \mathrm{TeV}$. As shown in Fig. 1, the DAMPE excess events are well fitted by the hard spectrum from a local DM subhalo if DM pair-annihilates into $e^{-} e^{+}$. For comparison, we also calculate the electron and positron flux from DM annihilations in the MW halo, which, however, is two orders of magnitude smaller and exhibits a rather flat spectrum over a much larger energy range.

In Fig. 2, we overlay signals from different DM models with the fourteen high energy bins in the DAMPE data. Instead of using the global-fitting background as in Fig. 1, a simple power law background $C E^{-\gamma}$ is employed for the $\mathrm{TeV}$ electrons. The best-fit PL has $C=2.4 \times 10^{4} \mathrm{GeV}^{2} \mathrm{~m}^{-2} \mathrm{~s}^{-1} \mathrm{sr}^{-1}$ and $\gamma=0.78$ with $\chi^{2}=20.4$, if we do not include DM contributions.

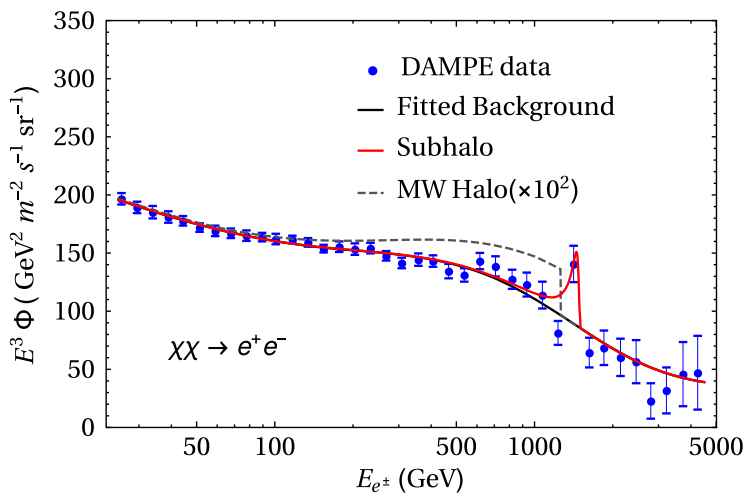

FIG. 1. The DAMPE electron data and the DM signals via $\chi \chi \rightarrow e^{+} e^{-}$. The black line represents the fitted background; the red line is the contribution from a local DM subhalo; the dashed gray line is the contribution from the Milky Way halo. $m_{\chi}=$ $1.5 \mathrm{TeV}$ and $\langle\sigma v\rangle=3 \times 10^{-26} \mathrm{~cm}^{3} / \mathrm{s}$.
Next, we consider DM contributions for three different annihilation modes: (A) $\chi \chi \rightarrow X \rightarrow e^{+} e^{-}$, (B) $\chi \chi \rightarrow$ $X X \rightarrow 2 e^{+} 2 e^{-},(C) \chi \chi \rightarrow X \rightarrow \bar{f} f$. In the case $A$ and $B$, the $X$ boson couples only electrons; in the case $C$, the $X$ boson couples to all SM fermions with universal couplings. For different DM annihilation channels, we employ $\Phi_{B}+F \Phi_{\chi}^{0}$ to fit the fourteen high energy data points, where $\Phi_{B}=C E^{-\gamma}, \Phi_{\chi}^{0}$ is the flux corresponding to the canonical thermal cross section $\sigma v=3 \times$ $10^{-26} \mathrm{~cm}^{3} / \mathrm{s}$, and $F$ is an overall floating parameter. The DM mass is fixed at $1.5 \mathrm{TeV}$ for the case $A$ and $C$, $3 \mathrm{TeV}$ for the case $B$. For the case $A$, the best-fit model has $C=4.7 \times 10^{4} \mathrm{GeV}^{2} \mathrm{~m}^{-2} \mathrm{~s}^{-1} \mathrm{sr}^{-1}, \quad \gamma=0.89$ and $F=1.03$, with $\chi^{2}=9.0$ which is improved by $\Delta \chi^{2}=11.4$ (hereafter $\Delta \chi^{2}$ denotes the improvement from the background-only PL fitting). In the case $B$, $\chi \chi \rightarrow X X \rightarrow 2 e^{+} 2 e^{-}$, the energy spectrum of electron and positron is a box-shaped distribution [24-29] which depends on the mass gap $\Delta m=m_{\chi}-M_{X}$ which should be sufficiently small to explain the sharp energy spectrum in the DAMPE data. We take $\Delta m=2 \mathrm{GeV}$ and $F=0.4$ which has $\chi^{2}=15.3\left(\Delta \chi^{2}=5.1\right)$. In the case $C$, $\chi \chi \rightarrow X \rightarrow \bar{f} f$, we used PPPC4DMID to compute the energy spectrum. In our model in the case $C$, the branching ratio $B R=1 / 24(1 / 8)$ for each lepton (quark) final state. We find that $\chi^{2}$ is $9.4\left(\Delta \chi^{2}=11\right)$ for $F=24$. The flux in the case $C$ overlaps with that in the case $A$ indicating that the electron channel is the dominant mode.

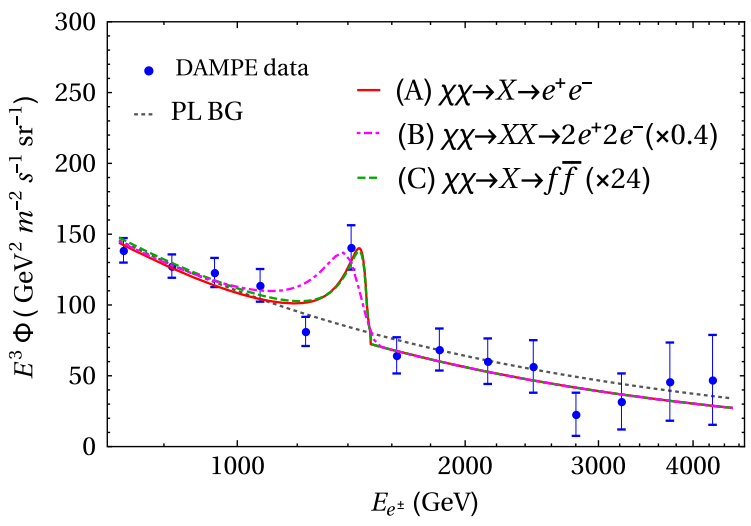

FIG. 2. The fourteen high energy bins in the DAMPE data and contributions from various DM models. The background is given by a simple power law $C E^{-\gamma}$ with $C=2.4(4.7) \times$ $10^{4} \mathrm{GeV}^{2} \mathrm{~m}^{-2} \mathrm{~s}^{-1} \mathrm{sr}^{-1}$ and $\gamma=0.78(0.89)$, for the background (background plus signal) fitting. The red solid, magenta dashdotted and green dashed lines correspond to the DM contributions from the processes $\chi \chi \rightarrow X \rightarrow e^{+} e^{-}, \chi \chi \rightarrow X X \rightarrow 2 e^{+} 2 e^{-}$, and $\chi \chi \rightarrow X \rightarrow \bar{f} f$ respectively. The annihilation cross section is $\langle\sigma v\rangle=3 \times 10^{-26} \mathrm{~cm}^{3} / \mathrm{s}$, and the DM mass is taken to be $1.5 \mathrm{TeV}$ for the $e^{+} e^{-}$and $\bar{f} f$ cases, $3 \mathrm{TeV}$ for the $4 e$ case. To generate the observed electron excess, an overall factor of 24 (0.4) is multiplied for the $\bar{f} f(4 e)$ channel. 


\section{COLLIDER CONSTRAINTS}

If the $X$ boson couples to both quarks and leptons, one can search for the $X$ boson resonance in the dilepton signal at the LHC. ATLAS collaboration also used the fourfermion contact interaction Lagrangian to interpret the most recent LHC results on dilepton signals [30]

$$
\mathcal{L}=\frac{4 \pi}{\Lambda^{2}} \eta_{i j}\left(\bar{q}_{i} \gamma^{\mu} q_{i}\right)\left(\bar{\ell}_{j} \gamma_{\mu} \ell_{j}\right)
$$

where $i, j=L, R$ for different chiral interactions, and $\eta_{i j}=$ \pm denotes constructive and destructive interferences with the SM processes. In our model, $\Lambda=\sqrt{4 \pi} M_{X} / \sqrt{\left|g_{q} g_{\ell}\right|}$. The most stringent $95 \%$ C.L. lower limit on $\Lambda$ analyzed using the recent ATLAS data [30] comes from the $\ell \ell$ final state for the $L L$ chiral interaction in the constructive case, $\Lambda>40 \mathrm{TeV}$, which gives rise to a constraint $\sqrt{\left|g_{q} g_{\ell}\right|}<0.09\left(M_{X} / \mathrm{TeV}\right)$.

The LEPII experiment also constrains the new physics models via the contact interaction operators. The LEPII group finds that $\Lambda_{V V}>15.9 \mathrm{TeV}$ in the $e^{-} e^{+} \rightarrow$ $e^{-} e^{+}$process at $95 \%$ C.L. [31,32]. The theoretical value of $\Lambda_{V V}$ predicted in our model is $\Lambda_{V V}=\sqrt{\pi} M_{X} / g_{e}$; thus the constraint on the electron coupling is given $g_{e} \lesssim 0.11\left(M_{X} / \mathrm{TeV}\right)$.

\section{DM DIRECT DETECTION CONSTRAINTS}

PandaX experiment [33] provides the best constraints to DM-proton spin-independent (SI) cross section for the $1.5 \mathrm{TeV} \mathrm{DM}, \quad \sigma_{\chi p}^{\mathrm{SI}} \lesssim 1.7 \times 10^{-45} \mathrm{~cm}^{2}$. The theoretical prediction of the SI DM-proton cross section in our model is $\sigma_{\chi p}=\mu_{\chi p}^{2} g_{\chi}^{2} g_{p}^{2} /\left(\pi M_{X}^{4}\right)$ where $\mu_{\chi p}$ is the reduced mass of the DM-proton system and $g_{p}=2 g_{u}+g_{d}$. The above SI cross section upper limit provides a constraint on the coupling $\sqrt{\left|g_{\chi} g_{p}\right|} \lesssim 6 \times 10^{-2}\left(M_{X} / \mathrm{TeV}\right)$.

If the $X$ boson only couples to the SM electrons, we need consider the DM direct detection limits due to electron recoils. Reference [34] analyzed the Xenon10 and Xenon100 results to constrain the interaction cross section between DM and electron; for the 1.5 TeV DM, the upper bound on the cross section between DM and the free electron is $\sigma_{\chi e}<3 \times 10^{-38} \mathrm{~cm}^{2}$. The theoretical prediction of the DM-electron cross section in our model is $\sigma_{\chi e}=\mu_{\chi e}^{2} g_{\chi}^{2} g_{e}^{2} /\left(\pi M_{X}^{4}\right)$. Thus the upper bound on the couplings is $\sqrt{\left|g_{\chi} g_{e}\right|} \lesssim 1.8 \times 10^{2}\left(M_{X} / \mathrm{TeV}\right)$.

To interpret the direct detection limit, we only assume the DM contribution from the MW halo. Although the DM density from the subhalo can be significant, its contribution to the DM direct detection signal is offset by the smaller velocity dispersion in the subhalo and the lack of the relative motion with respect to Earth, since the subhalo is also assumed to rotate around the Galactic Center.

\section{DM INDIRECT DETECTION CONSTRAINTS}

For the $1.5 \mathrm{TeV} \mathrm{DM}$ annihilating into two SM particles, H.E.S.S. data [35] constrain the annihilation cross sections: $\langle\sigma v\rangle<2(6) \times 10^{-26} \mathrm{~cm}^{3} / \mathrm{s}$ for the $\tau^{+} \tau^{-}\left(W^{+} W^{-}\right)$channel, which are based on 10-year data of the inner $300 \mathrm{pc}$ of the Galactic Center region assuming the Einasto profile. This provides a very strong constraint on the case where the $X$ boson couple universally to all SM fermions.

For the pair-annihilation case, $\chi \chi \rightarrow X X$, the most stringent constraint comes from the H.E.S.S. data [36,35], which is stronger than the limits from the Fermi-LAT data in the direction of the dwarf spheroidal galaxies [37] and from the Planck CMB data which is sensitive to energy injection to the CMB from DM annihilations $[38,39]$. The H.E.S.S. limit in the $\chi \chi \rightarrow 4 e$ channel is $\langle\sigma v\rangle<6(20) \times 10^{-25} \mathrm{~cm}^{3} / \mathrm{s}$ in the $m_{\chi} \sim M_{X}$ $\left(m_{\chi} \gg M_{X}\right)$ case, for the $3 \mathrm{TeV}$ DM. The required DM annihilation cross section for the DAMPE excess in the $4 e$ case is consistent with the above H.E.S.S. constraint.

The IceCube experiment [40] sets an upper bound on the DM annihilation cross section for various SM channels by analyzing the neutrino signal $\langle\sigma v\rangle<\mathcal{O}(1) \times 10^{-22} \mathrm{~cm}^{3} / \mathrm{s}$, which is much larger than the annihilation cross section needed for the DAMPE excess.

\section{PARAMETER SPACE}

For the electrophilic case in which the $X$ boson only couples to the electrons, to satisfy the LEPII and direct detection constraints, we select a benchmark model point as follows $\left(\delta m, g_{e}, g_{\chi}\right)=(100 \mathrm{GeV}, 0.1,0.4)$ where $\delta m \equiv$ $2 m_{\chi}-M_{X}$. The DM annihilation for this benchmark model is $\sigma v \simeq 1.3 \mathrm{pb}$ which can explain the DAMPE excess and the relic density.

For the universal case in which the $X$ boson couples to all SM fermions with equal coupling strength, we select a benchmark model point as follows $\left(\delta m, g_{f}, g_{\chi}\right)=$ $\left(10 \mathrm{GeV}, 4 \times 10^{-3}, 1\right)$. The DM annihilation at the halo is $\sigma v \simeq 30 \mathrm{pb}$; the DM annihilation at $v=1 / 4$ (the typical velocity for DM thermal freeze-out) is $\sigma v \simeq 0.3 \mathrm{pb}$ which is smaller than $1 \mathrm{pb}$. However, since the annihilation cross section depends on the velocity of the DM, one has to take into account the thermal average at the freeze-out. Such an enhancement of the halo annihilation was studied previously in the context of the PAMELA positron excess [41-43]. Figure 3 shows the relic density line and the ATLAS and PandaX constraints for the universal $X$ case near the resonance region. The benchmark model point is consistent with all constraints as shown in Fig. 3. The universal case is in tension with the H.E.S.S. constraint for the $\tau^{+} \tau^{-}$channel with an Einasto profile. However, for a different DM profile, the tension with the H.E.S.S. constraint could be alleviated.

For the case where DM annihilates into a pair of on-shell $X$ bosons, since the coupling between the $X$ boson and the 


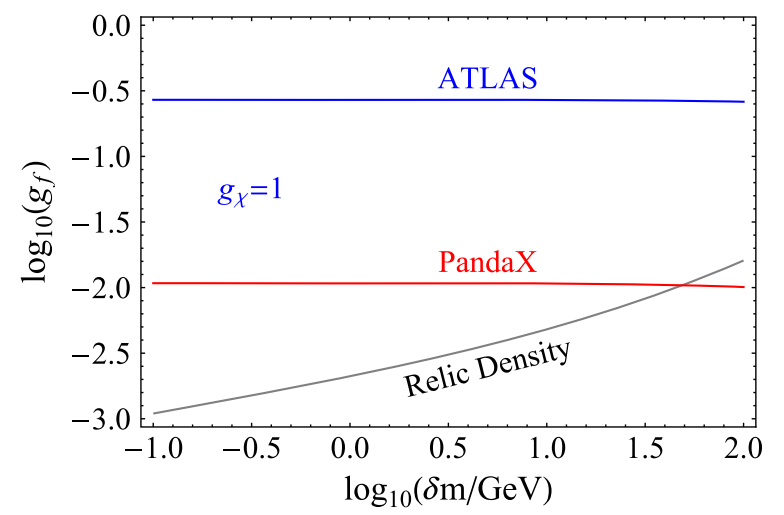

FIG. 3. Constraints on the parameter space $\left(\delta m, g_{f}\right)$ for the $g_{\chi}=1$ case. The blue and red lines correspond to the ATLAS and PandaX upper bounds; the black line indicates the parameter space that can generate the correct DM relic density.

SM particles can be significantly small, the only relevant constraint comes from the indirect detection limits. In our case, the most stringent constraint comes from the H.E.S.S. data, which, however, is one order of magnitude higher than the thermal annihilation cross section. In order to produce a narrow energy spectrum for the injection source function in this case, the mass difference between the DM and the $X$ boson has to be very small, which compress the phase space of the DM annihilation so that $\sigma v(\chi \chi \rightarrow X X)$ is very small for perturbative $g_{\chi}$ coupling. Thus one has to be in the nonperturbative region of the parameter space to generate a large annihilation cross section and a narrow energy spectrum for this scenario.

\section{CONCLUSIONS}

We have proposed a simple dark matter model to explain the high energy electron excess events recently observed by the DAMPE experiment. The morphology of the energy spectrum of the excess events hints a local source for the high energy electrons. We investigated the possibility of the DM annihilations in a local subhalo which is $0.3 \mathrm{kpc}$ away from us to generate such an excess.

Three scenarios in the model were investigated. The case where DM only annihilates into $e^{+} e^{-}$provides a good fitting to the excess while satisfying the various constraints. In the case where the $X$ boson couples universally with all SM fermions, DM has to annihilate near the $X$ boson resonance to generate a much larger annihilation cross section to explain the excess. In the case where DM annihilates into on-shell $X$ bosons, in order to produce the sharp excess in the energy spectrum, the mass gap between the DM and the $X$ boson has to be in the $\mathrm{GeV}$ range.

\section{ACKNOWLEDGMENTS}

We thank Lei Feng, Yue-Lin Sming Tsai, Qiang Yuan, Cun Zhang for helpful correspondence or discussions. The work is supported in part by the National Natural Science Foundation of China under Grant Nos. 11775109 and U1738134, by the National Recruitment Program for Young Professionals, by the Nanjing University Grant No. 14902303, by the National Postdoctoral Program for Innovative Talents under Grant No. BX201700116, and by the Jiangsu Planned Projects for Postdoctoral Research Funds under Grant No. 1701130B.

Note added.-After the submission of the first version of our paper on arXiv, a number of papers appeared to interpret the DAMPE anomaly, including astrophysical explanations [44,45] and DM studies [45-72]. The conclusions in these DM papers are consistent with ours.
[1] G. Ambrosi et al. (DAMPE Collaboration), Direct detection of a break in the teraelectronvolt cosmic-ray spectrum of electrons and positrons, Nature (London) 552, 63 (2017).

[2] L. Accardo et al. (AMS Collaboration), High Statistics Measurement of the Positron Fraction in Primary Cosmic Rays of 0.5-500 GeV with the Alpha Magnetic Spectrometer on the International Space Station, Phys. Rev. Lett. 113, 121101 (2014).

[3] M. Aguilar et al. (AMS Collaboration), Electron and Positron Fluxes in Primary Cosmic Rays Measured with the Alpha Magnetic Spectrometer on the International Space Station, Phys. Rev. Lett. 113, 121102 (2014).

[4] M. Aguilar et al. (AMS Collaboration), Precision Measurement of the $\left(e^{+}+e^{-}\right)$Flux in Primary Cosmic Rays from $0.5 \mathrm{GeV}$ to $1 \mathrm{TeV}$ with the Alpha Magnetic
Spectrometer on the International Space Station, Phys. Rev. Lett. 113, 221102 (2014).

[5] S. Abdollahi et al. (Fermi-LAT Collaboration), Cosmic-ray electron-positron spectrum from $7 \mathrm{GeV}$ to $2 \mathrm{TeV}$ with the Fermi Large Area Telescope, Phys. Rev. D 95, 082007 (2017).

[6] O. Adriani et al. (CALET Collaboration), Energy Spectrum of Cosmic-Ray Electron and Positron from $10 \mathrm{GeV}$ to $3 \mathrm{TeV}$ Observed with the Calorimetric Electron Telescope on the International Space Station, Phys. Rev. Lett. 119, 181101 (2017).

[7] J. Chang et al. (DAMPE Collaboration), The Dark Matter Particle Explorer mission, Astropart. Phys. 95, 6 (2017).

[8] Y.Z. Fan, W. C. Huang, M. Spinrath, Y. L. S. Tsai, and Q. Yuan, A model explaining neutrino masses and the 
DAMPE cosmic ray electron excess, Phys. Lett. B 781, 83 (2018).

[9] G. H. Duan, L. Feng, F. Wang, L. Wu, J. M. Yang, and R. Zheng, Simplified TeV leptophilic dark matter in light of DAMPE data, J. High Energy Phys. 02 (2018) 107.

[10] P. H. Gu and X. G. He, Electrophilic dark matter with dark photon: From DAMPE to direct detection, Phys. Lett. B 778, 292 (2018).

[11] Q. Yuan et al., Interpretations of the DAMPE electron data, arXiv:1711.10989.

[12] K. Fang, X. J. Bi, and P. F. Yin, Explanation of the knee-like feature in the DAMPE cosmic $e^{-}+e^{+}$energy spectrum, Astrophys. J. 854, 57 (2018).

[13] M. Cirelli, R. Franceschini, and A. Strumia, Minimal dark matter predictions for galactic positrons, anti-protons, photons, Nucl. Phys. B800, 204 (2008).

[14] V. L. Ginzburg and S. I. Syrovatskii, The Origin of Cosmic Rays (Pergamon, Oxford, 1964).

[15] M. Kuhlen and D. Malyshev, ATIC, PAMELA, HESS, Fermi and nearby dark matter subhalos, Phys. Rev. D 79, 123517 (2009).

[16] T. Delahaye, J. Lavalle, R. Lineros, F. Donato, and N. Fornengo, Galactic electrons and positrons at the Earth:new estimate of the primary and secondary fluxes, Astron. Astrophys. 524, A51 (2010).

[17] J. F. Navarro, C. S. Frenk, and S. D. M. White, A universal density profile from hierarchical clustering, Astrophys. J. 490, 493 (1997).

[18] J. Choquette, Constraining dwarf spheroidal dark matter halos with the galactic center excess, Phys. Rev. D 97, 043017 (2018).

[19] M. G. Walker, M. Mateo, and E. Olszewski, Stellar velocities in the Carina, Fornax, Sculptor and Sextans dSph Galaxies: Data from the Magellan/MMFS survey, Astron. J. 137, 3100 (2009).

[20] J. D. Simon and M. Geha, The kinematics of the ultra-faint Milky Way satellites: Solving the missing satellite problem, Astrophys. J. 670, 313 (2007).

[21] X. Huang, Y. L. S. Tsai, and Q. Yuan, LikeDM: Likelihood calculator of dark matter detection, Comput. Phys. Commun. 213, 252 (2017).

[22] M. Cirelli, G. Corcella, A. Hektor, G. Hütsi, M. Kadastik, P. Panci, M. Raidal, F. Sala, and A. Strumia, PPPC 4 DM ID: A poor particle physicist cookbook for dark matter indirect detection, J. Cosmol. Astropart. Phys. 03 (2011) 051; Erratum, 10 (2012) E01.

[23] P. Ciafaloni, D. Comelli, A. Riotto, F. Sala, A. Strumia, and A. Urbano, Weak corrections are relevant for dark matter indirect detection, J. Cosmol. Astropart. Phys. 03 (2011) 019.

[24] A. Ibarra, S. Lopez Gehler, and M. Pato, Dark matter constraints from box-shaped gamma-ray features, J. Cosmol. Astropart. Phys. 07 (2012) 043.

[25] J. Mardon, Y. Nomura, D. Stolarski, and J. Thaler, Dark matter signals from cascade annihilations, J. Cosmol. Astropart. Phys. 05 (2009) 016.

[26] M. Abdullah, A. DiFranzo, A. Rajaraman, T. M. P. Tait, P. Tanedo, and A. M. Wijangco, Hidden on-shell mediators for the Galactic Center $\gamma$-ray excess, Phys. Rev. D 90, 035004 (2014).
[27] J. M. Cline, G. Dupuis, Z. Liu, and W. Xue, The windows for kinetically mixed Z'-mediated dark matter and the galactic center gamma ray excess, J. High Energy Phys. 08 (2014) 131.

[28] P. Agrawal, B. Batell, P. J. Fox, and R. Harnik, WIMPs at the Galactic Center, J. Cosmol. Astropart. Phys. 05 (2015) 011.

[29] J. M. Cline, G. Dupuis, Z. Liu, and W. Xue, Multimediator models for the galactic center gamma ray excess, Phys. Rev. D 91, 115010 (2015).

[30] M. Aaboud et al. (ATLAS Collaboration), Search for new high-mass phenomena in the dilepton final state using $36 \times \mathrm{fb}^{-1}$ of proton-proton collision data at $s \sqrt{=13}$ $\mathrm{TeV}$ with the ATLAS detector, J. High Energy Phys. 10 (2017) 182.

[31] t. S. Electroweak (LEP and ALEPH and DELPHI and L3 and OPAL Collaborations and LEP Electroweak Working Group and SLD Electroweak Group and SLD Heavy Flavor Group Collaboration), A combination of preliminary electroweak measurements and constraints on the standard model, arXiv:hep-ex/0312023.

[32] S. Schael et al. (ALEPH Collaboration), Fermion pair production in $e^{+} e^{-}$collisions at $189-209-\mathrm{GeV}$ and constraints on physics beyond the standard model, Eur. Phys. J. C 49, 411 (2007).

[33] X. Cui et al. (PandaX-II Collaboration), Dark Matter Results From 54-Ton-Day Exposure of PandaX-II Experiment, Phys. Rev. Lett. 119, 181302 (2017).

[34] R. Essig, T. Volansky, and T. T. Yu, New constraints and prospects for sub-GeV dark matter scattering off electrons in xenon, Phys. Rev. D 96, 043017 (2017).

[35] H. Abdallah et al. (H.E.S.S. Collaboration), Search for Dark Matter Annihilations towards the Inner Galactic Halo from 10 years of Observations with H.E.S.S, Phys. Rev. Lett. 117, 111301 (2016).

[36] S. Profumo, F. S. Queiroz, J. Silk, and C. Siqueira, Searching for secluded dark matter with H.E.S.S., Fermi-LAT, and Planck, arXiv:1711.03133.

[37] M. Ackermann et al. (Fermi-LAT Collaboration), Searching for Dark Matter Annihilation from Milky Way Dwarf Spheroidal Galaxies with Six Years of Fermi Large Area Telescope Data, Phys. Rev. Lett. 115, 231301 (2015).

[38] T. R. Slatyer, Indirect dark matter signatures in the cosmic dark ages. I. Generalizing the bound on s-wave dark matter annihilation from Planck results, Phys. Rev. D 93, 023527 (2016).

[39] T. R. Slatyer, Indirect dark matter signatures in the cosmic dark ages II. Ionization, heating and photon production from arbitrary energy injections, Phys. Rev. D 93, 023521 (2016).

[40] M. G. Aartsen et al. (IceCube Collaboration), Search for neutrinos from dark matter self-annihilations in the center of the Milky Way with 3 years of IceCube/DeepCore, Eur. Phys. J. C 77, 627 (2017).

[41] D. Feldman, Z. Liu, and P. Nath, PAMELA positron excess as a signal from the hidden sector, Phys. Rev. D 79, 063509 (2009).

[42] M. Ibe, H. Murayama, and T. T. Yanagida, Breit-Wigner enhancement of dark matter annihilation, Phys. Rev. D 79, 095009 (2009). 
[43] W. L. Guo and Y.L. Wu, Enhancement of dark matter annihilation via Breit-Wigner resonance, Phys. Rev. D 79, 055012 (2009).

[44] I. Cholis, T. Karwal, and M. Kamionkowski, Features in the spectrum of cosmic-ray positrons from pulsars, Phys. Rev. D 97, 123011 (2018).

[45] X. J. Huang, Y. L. Wu, W. H. Zhang, and Y. F. Zhou, Origins of sharp cosmic-ray electron structures and the DAMPE excess, Phys. Rev. D 97, 091701 (2018).

[46] L. Zu, C. Zhang, L. Feng, Q. Yuan, and Y.Z. Fan, Constraints on box-shaped cosmic ray electron feature from dark matter annihilation with the AMS-02 and DAMPE data, arXiv:1711.11052.

[47] Y. L. Tang, L. Wu, M. Zhang, and R. Zheng, Lepton-portal dark matter in hidden valley model and the DAMPE recent results, arXiv:1711.11058.

[48] W. Chao and Q. Yuan, The electron-flavored Z'-portal dark matter and the DAMPE cosmic ray excess, arXiv:1711 .11182 .

[49] P. Athron, C. Balazs, A. Fowlie, and Y. Zhang, Modelindependent analysis of the DAMPE excess, J. High Energy Phys. 02 (2018) 121.

[50] J. Cao, L. Feng, X. Guo, L. Shang, F. Wang, and P. Wu, Scalar dark matter interpretation of the DAMPE data with U(1) gauge interactions, arXiv:1711.11452.

[51] G. H. Duan, X. G. He, L. Wu, and J. M. Yang, Leptophilic dark matter in gauged $U(1)_{L_{e}-L_{\mu}}$ model in light of DAMPE cosmic ray $e^{+}+e^{-}$excess, Eur. Phys. J. C 78, 323 (2018).

[52] P. H. Gu, Radiative Dirac neutrino mass, DAMPE dark matter and leptogenesis, arXiv:1711.11333.

[53] W. Chao, H. K. Guo, H. L. Li, and J. Shu, Electron flavored dark matter, Phys. Lett. B 782, 517 (2018).

[54] C. H. Chen, C. W. Chiang, and T. Nomura, Explaining the DAMPE $e^{+} e^{-}$excess using the Higgs triplet model with a vector dark matter, Phys. Rev. D 97, 061302 (2018).

[55] T. Li, N. Okada, and Q. Shafi, Scalar dark matter, Type II Seesaw and the DAMPE cosmic ray $e^{+}+e^{-}$excess, Phys. Lett. B 779, 130 (2018).

[56] R. Zhu and Y. Zhang, Graviton-mediated dark matter model explanation the DAMPE electron excess and search at $e^{+} e^{-}$ colliders, arXiv:1712.01143.

[57] P. H. Gu, Quasi-degenerate dark matter for DAMPE excess and $3.5 \mathrm{keV}$ line, arXiv:1712.00922.

[58] T. Nomura and H. Okada, Radiative seesaw models linking to dark matter candidates inspired by the DAMPE excess, Phys. Dark Universe 21, 90 (2018).
[59] K. Ghorbani and P. H. Ghorbani, DAMPE electron-positron excess in leptophilic $Z^{\prime}$ model, J. High Energy Phys. 05 (2018) 125.

[60] J. Cao, L. Feng, X. Guo, L. Shang, F. Wang, P. Wu, and L. $\mathrm{Zu}$, Explaining the DAMPE data with scalar dark matter and gauged $U(1)_{L_{e}-L_{\mu}}$ interaction, Eur. Phys. J. C 78, 198 (2018).

[61] J. S. Niu, T. Li, R. Ding, B. Zhu, H. F. Xue, and Y. Wang, Bayesian analysis of the DAMPE lepton spectra and two simple model interpretations, Phys. Rev. D 97, 083012 (2018).

[62] F. Yang, M. Su, and Y. Zhao, Dark matter annihilation from nearby ultra-compact micro halos to explain the tentative excess at 1.4 TeV in DAMPE data, arXiv:1712.01724.

[63] R. Ding, Z. L. Han, L. Feng, and B. Zhu, Neutrino and dark matter confronting DAMPE excess, Chin. Phys. C 42, 083104 (2018).

[64] G. L. Liu, F. Wang, W. Wang, and J. M. Yang, Explaining DAMPE results by dark matter with hierarchical leptonspecific Yukawa interactions, Chin. Phys. C 42, 035101 (2018).

[65] S. F. Ge, H. J. He, and Y. C. Wang, Flavor structure of the cosmic-ray electron/positron excesses at DAMPE, Phys. Lett. B 781, 88 (2018).

[66] Y. Zhao, K. Fang, M. Su, and M. C. Miller, A strong test of the dark matter origin of the 1.4 TeV DAMPE signal using IceCube neutrinos, J. Cosmol. Astropart. Phys. 06 (2018) 030 .

[67] Y. Sui and Y. Zhang, Prospects of type-II seesaw at future colliders in light of the DAMPE $e^{+} e^{-}$excess, Phys. Rev. D 97, 095002 (2018).

[68] N. Okada and O. Seto, DAMPE excess from decaying righthanded neutrino dark matter, arXiv:1712.03652.

[69] J. Cao, X. Guo, L. Shang, F. Wang, P. Wu, and L. Zu, Scalar dark matter explanation of the DAMPE data in the minimal Left-Right symmetric model, Phys. Rev. D 97, 063016 (2018).

[70] Z. L. Han, W. Wang, and R. Ding, Radiative seesaw model and DAMPE excess from leptophilic gauge symmetry, Eur. Phys. J. C 78, 216 (2018).

[71] J.S. Niu, T. Li, and F. Z. Xu, The simple and natural interpretations of the DAMPE cosmic ray electron/positron spectrum within two sigma deviations, arXiv:1712.09586.

[72] T. Nomura, H. Okada, and P. Wu, A radiative neutrino mass model in light of DAMPE excess with hidden gauged $U(1)$ symmetry, J. Cosmol. Astropart. Phys. 05 (2018) 053. 